\title{
Opportunities for negative ions studies at the Frankfurt Low-energy Storage Ring (FLSR)
}

\section{Oliver Forstner ${ }^{1,2}$ (1) $\cdot$ Jan Müller ${ }^{3} \cdot$ Kurt Ernst Stiebing ${ }^{3}$}

Published online: 22 April 2020

(C) The Author(s) 2020

\begin{abstract}
The room-temperature electrostatic heavy ion storage ring FLSR was originally designed to study the collision dynamics of atoms and molecules. Recently it has been equipped with a RF plasma ion source combined with a charge exchange cell to be able to perform studies with negative ions in the ring. In preliminary experiments beams of $\mathrm{He}^{-}, \mathrm{O}^{-}$and $\mathrm{OH}^{-}$could successfully be stored. The measured lifetime of the metastable $\mathrm{He}^{-}$-ion is in good agreement with previous results, showing that the lifetime measurement in this case is not limited by the storage time due to collisional detachment. In the case of $\mathrm{O}^{-}$and $\mathrm{OH}^{-}$ storage times in the order of seconds have been achieved. In a next step laser beams will be introduced in the ring allowing photodetachment studies of vibrationally cold molecules.
\end{abstract}

Keywords Negative ions · Electrostatic storage ring $\cdot$ Laser photodetachment

\section{Introduction}

Negative ions are fragile quantum systems where an extra electron is weakly bound to the neutral atom or molecule due to strong electron-electron correlation effects. The binding energy (the so-called electron affinity EA) is usually less than a few eV with a potential which asymptotically decays as $r^{-4}$. In general, this allows only one bound state with a handful of exceptions featuring an exiting state (notably $\mathrm{Si}^{-}$exhibiting two exited states). A compilation of the EA of atomic negative ions can be found e.g. in [1]. As the EA of most negative ions correspond to wavelength in the VIS or NIR spectral photon range this makes studying the properties of negative ions with lasers highly attractive. Tuneable laser

This article is part of the Topical Collection on Proceedings of PLATAN 2019, 1st International Conference, Merger of the Poznan Meeting on Lasers and Trapping Devices in Atomic Nuclei Research and the International Conference on Laser Probing, Mainz, Germany 19-24 May 2019

Edited by Krassimira Marinova, Michael Block, Klaus D.A. Wendt and Magdalena Kowalska

Oliver Forstner

oliver.forstner@uni-jena.de

1 Friedrich-Schiller-Universität Jena, Jena, Germany

2 Helmholtz Institute Jena, Jena, Germany

3 Institut für Kernphysik (IKF), Goethe University, Frankfurt, Germany 
systems with $\mathrm{cw}$ or high repetition rates of $10 \mathrm{kHz}$ and above with laser intensities of some Watt allows precise determination of the EA by using Laser Photodetachment Threshold (LPT) spectroscopy. There the photon energy is scanned around the threshold and the onset of neutralisation of the negative ion is measured. In the case of negative molecular ions the situation is complex due to the internal degrees of freedom of the molecule (rotation, vibration,...). The internal energy of the molecule adds to the photon energy leading to a reduction of the measured threshold. The usual way of producing negative ions in either a Middleton type sputter ion source [2] or by double electron capture in a charge exchange chamber exhibiting alkali metal vapour (see e.g. [3]) produces beams of "hot" negative molecular ions. It is therefore necessary to reduce the internal energy of the negative molecular ion before performing LPT studies. A storage ring is an ideal tool for such studies, as the stored ion can be allowed to relax to a lower vibrational or rotational state before performing the LPT measurement. In an ideal situation, the molecule can relax down to the ambient temperature of the instrument. This limits the use of a room-temperature device in its lowest reachable state before a re-heating by black-body radiation takes place. However, at least the vibrational ground state can usually be reached. The rotational lowest states can only be reached in a cryogenic storage ring like CSR@Heidelberg [4] or DESIREE@ Stockholm [5], where the whole storage ring is cooled to only a few Kelvin above absolute zero.

Negative ions play an important role in accelerator mass spectrometry (AMS). In AMS mainly tandem accelerators are used as acceleration device which makes the use of negative ions at the injector side indispensable. They further play a key role in isobar separation in AMS due to the element selectivity of the electron correlation. A notable example is radiocarbon dating, where the stable isobar of the radioactive ${ }^{14} \mathrm{C},{ }^{14} \mathrm{~N}$, does not form negative ions and is therefore not extracted from the ion source. In recent projects for selective isobar cleaning with the use of lasers in AMS laser photodetachment is used to selectively neutralise unwanted isobars already at the low energy side of an AMS machine (see e.g. [6] and the references therein). For these studies precise knowledge about the EAs of atomic and molecular negative ions is required. However, for the mass-range of the isotope of interest between $\mathrm{A}=50$ and $\mathrm{A}=200$ little or nothing is known about the EAs of corresponding molecular negative ions used for selective laser photodetachment. More experimental data of "cold" negative molecular ions is required which can easily be obtained from a room-temperature electrostatic storage ring like FLSR.

\section{Experimental setup}

The Frankfurt Low-energy Storage Ring (FLSR) is a small room-temperature electrostatic storage ring for ions up to $80 \mathrm{keV}$ at the Institut für Kernphysik (IKF) in Frankfurt [7]. It resembles a racetrack geometry with a circumference of the closed orbit of $14.23 \mathrm{~m}$. A sketch of the FLSR setup is shown in Fig. 1. An important parameter for studying negative ions is the residual gas pressure inside a storage ring. At energies of the order of some tens of $\mathrm{keV}$ the cross-section for collisional detachment is dominated by the geometrical cross-section for a collision between the ion and a residual gas molecule. Therefore the collisional detachment rate is inversely proportional to the residual gas pressure. The typical pressure in FLSR is $p_{F L S R} \approx 1.0-2.0 \times 10^{-10}$ mbar, corrected for $\mathrm{H}_{2}$ as residual gas. The injector of FLSR includes a high voltage platform where different ion sources can be mounted. For experiments with positive ions a Penning ion source was installed. To study negative ions a commercial RF plasma ion source with an rubidium charge exchange cell from National Electrostatics Corporation (NEC) was installed. In this type of ion source gas 

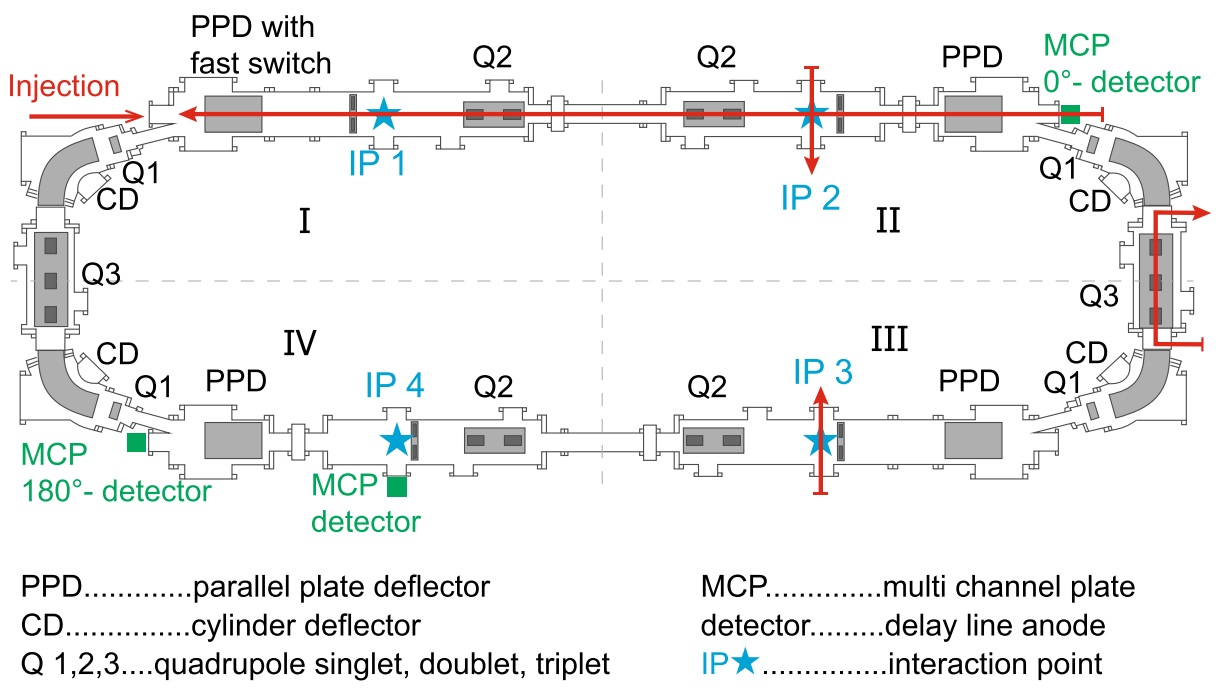

Fig. 1 Sketch of the FLSR with the main ion-optical components depicted. The four points of highest ion density are marked with stars. The possible positions for laser beams are marked with arrows. Both co-linear and transverse overlappings are possible. A discussion about this is given in Section 4

can be introduced into a plasma cell where singly charged positive ions are produced. These are accelerated by an electrostatic field and guided through a cell with rubidium vapour where a double electron capture leading to singly negatively charged ions can occur. It was designed for injection of $\mathrm{He}^{-}$ions into a tandem accelerator with intensities up to $4 \mu \mathrm{A}$ of beam current (hence the name Alphatross). For the experiments with negative ions a beam energy after the ion source of $20 \mathrm{keV}$ has been chosen. After leaving the ion source the ions are mass-selected with the help of an $90^{\circ}$ sector magnet. Before entering the FLSR the cw-beam is bunched by an electrostatic chopper. The length of the bunch is set to be about $80 \%$ of the revolution time of the ions in the ring, which depends on the mass and kinetic energy of the ions. This ensures, that the parallel plate deflector at the injector of the ring is switched to storage mode without ions passing through. The ring is operated in a coasting beam mode, i.e. the circulating bunch will disperse after some revolutions and eventually fill the entire ring with ions. At the end of both long sides of the ring position sensitive MCP detectors have been installed. These detectors measure the neutral particles travelling unaffected of the parallel plate deflectors mounted in front. In this way, neutral particles from either collisional detached or from laser photodetached ions can be registered. The detectors can be gated to the laser pulses, which increases the ratio between laser and collisional detachment and therefore the signal-to-noise ratio.

\section{Beam lifetime measurements}

The first steps after installation of the ion source was to show injection into the ring. After successfully storing negative ions in the ring the beam lifetime in the ring has been measured. To this end, neutral particles from collisional detachment of the ions with residual gas particles have been measured with the MCP detector at the straight section. Under stable conditions of the residual gas pressure the intensity of this signal is proportional to the 


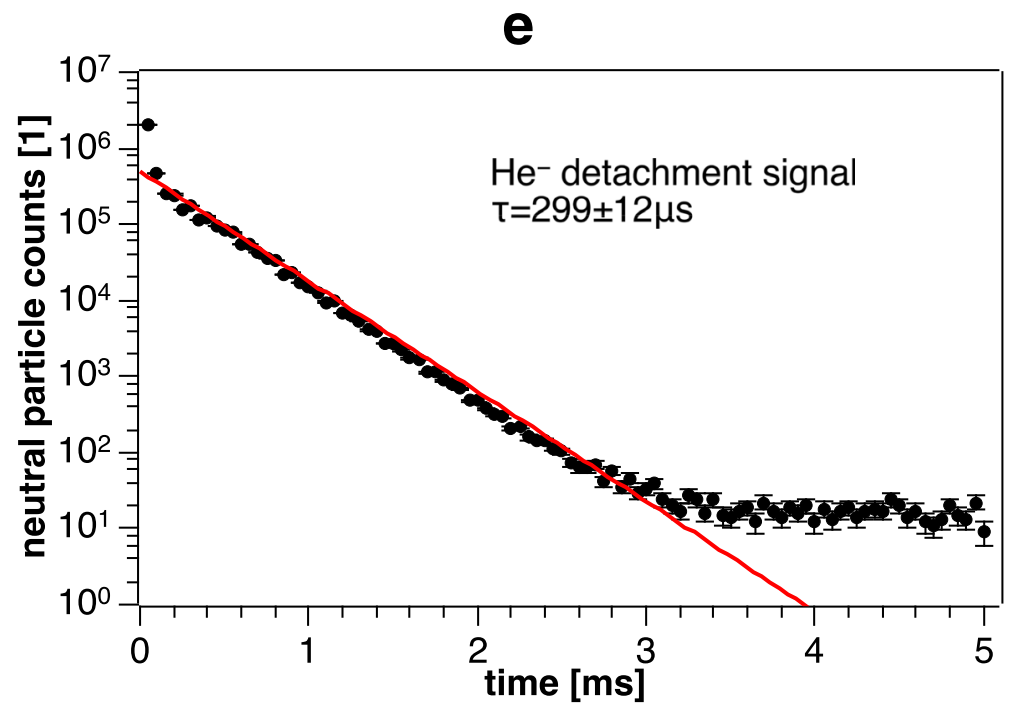

Fig. $2 \mathrm{He}^{-}$lifetime in the ring. The solid red line represents an exponential decay fit to the measured datapoints giving a lifetime of $299 \pm 12 \mu$ s. The deviation of the exponential decay towards the end of the curve represents the background resulting from dark counts in the detector. The deviation in the beginning of the curve results from ions which are not perfectly matched to the acceptance of the ring and therefore lost after a few revolutions. The measured lifetime is in good agreement with the previous results in the ELISA storage ring. This gives rise to the assumption, that the lifetime is not limited by the storage time in the ring

number of stored ions. As the Alphatross ion source was designed to produce intense beams of $\mathrm{He}^{-}$, this metastable negative ion was the natural choice to start with. The intrinsic lifetime of the $1 s 2 s 2 p^{4} P_{5 / 2}$ level of $\mathrm{He}^{-}$has been measured to be $365 \pm 3 \mu$ s in the electrostatic storage ring ELISA in Aarhus [8]. This value has already been corrected for the blackbodyradiation detachment of the "warm" storage ring. At room-temperature the lifetime has been measured to be $306.1 \pm 2.0 \mu \mathrm{s}$. This is in good agreement with the lifetime determined in FLSR (see Fig. 2). This clearly shows, that the storage time was actually much longer than the lifetime of $\mathrm{He}^{-}$in the ring and not limited by collisional detachment with residual gas particles. To determine the actual beam lifetime $\mathrm{O}^{-}$and $\mathrm{OH}^{-}$have been stored. The corresponding beam current at injection was $260 \mathrm{nA}$ for $\mathrm{O}^{-}$and $110 \mathrm{nA}$ for $\mathrm{OH}^{-}$. The $\mathrm{OH}^{-}$ ions have been produced by mixing $\mathrm{O}_{2}$ and $\mathrm{H}_{2}$ gas in the plasma ion source. The measured storage times for $\mathrm{O}^{-}$and $\mathrm{OH}^{-}$are 1.946(12) s and 0.843(4) s, respectively (Fig. 3). The uncertainty given is a combination of the counting statistics of the neutral particles and the fit to a pure exponential decay. This is well within the range of the theoretical predicted storage time based on the geometric cross-section for collisions with residual gas $\mathrm{H}_{2}$ molecules at a pressure of $1.0-2.0 \times 10^{-10} \mathrm{mbar}$ (corresponding to $4.0-2.0 \mathrm{~s}$ ). The shorter storage time of the molecular ion is attributed to the larger geometrical cross-section for collisions with residual gas particles.

\section{Discussion and outlook}

We have demonstrated the ability of FLSR to store negative atomic and molecular ions at energies of $20 \mathrm{keV}$. The known intrinsic lifetime of $\mathrm{He}^{-}$could well be reproduced in our 
e

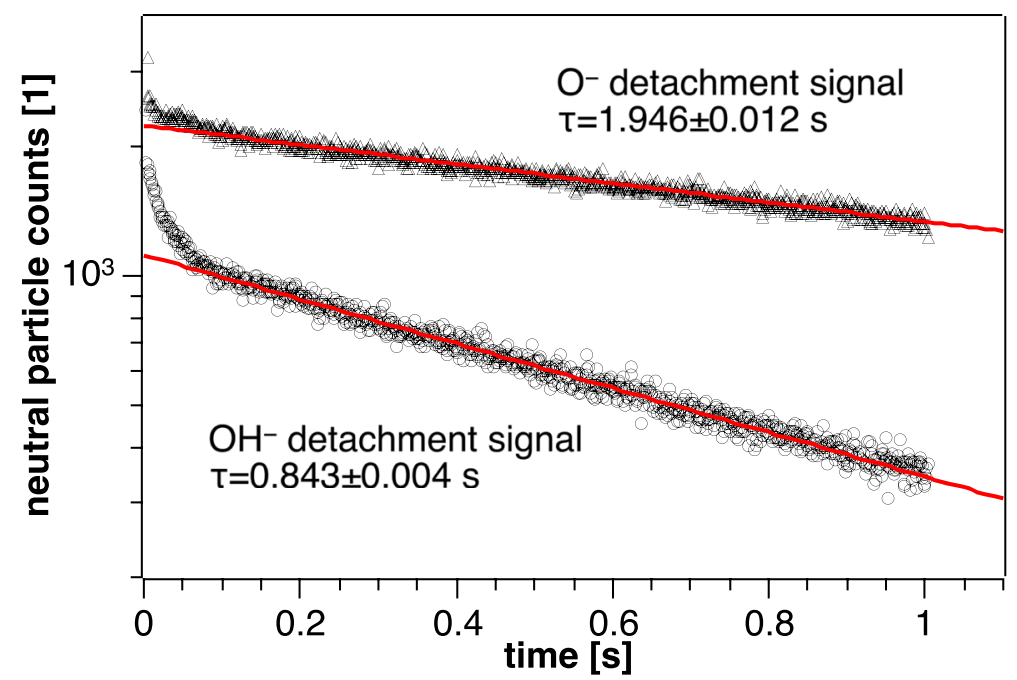

Fig. 3 Storage times of $\mathrm{O}^{-}$and $\mathrm{OH}^{-}$in the ring. The storage time is mainly limited due to collisions with residual gas atoms in the ring. The solid red lines represents an exponential decay fit to the measured datapoints. Similar to Fig. 2 the deviation from the exponential decay at the beginning of the curve results from ions not matched to the ring acceptance and therefore not used for the determination of the storage time. The molecular ion has a larger cross-section for collisions with residual gas molecules resulting in a reduced storage time

measurements and storage times in the order of seconds could be shown for the atomic ion $\mathrm{O}^{-}$and for the molecule $\mathrm{OH}^{-}$. Comparison of the measured storage times with estimates based on the residual gas pressure show, that collisions with residual gas molecules (mainly $\mathrm{H}_{2}$ ) are the dominating factor limiting the storage time of negative ions in FLSR. However, the achieved storage times are sufficient for studying laser photodetachment in the ring. The ions perform several ten thousand revolutions and the neutral particles resulting from collisional detachment can be discriminated by gating the detectors to the time structure of a pulsed laser. To perform these studies, laser grade windows for the VIS and NIR range have been installed at different positions (mainly around interaction point IP 2 in Fig. 1). In a next step a tuneable Ti:sapphire laser pumped by a frequency doubled Nd:YAG will be installed in the experimental hall. This will allow to study the photodetachment of $\mathrm{OH}^{-}$ around its threshold. Two different geometries for overlapping the laser and ion beam are under discussion: collinear or transverse. The collinear geometry has the disadvantage of the laser beam directly hitting the MCP detector and the necessary doppler correction of the photon energy with respect to the ion beam energy. Both problems can be avoided by using a transverse overlap in the interaction point IP 2. This comes at the cost of a much smaller interaction volume. However, the region of high particle density is limited to a small region around the four interaction points in FLSR. The gain of using the collinear geometry is therefore limited.

The currently installed Alphatross source is limited in the available ion species as only gaseous material can be introduced into the RF cell. Installation of a Middleton type sputter ion source is foreseen for the future. 
Funding Information Open Access funding provided by Projekt DEAL.

Open Access This article is licensed under a Creative Commons Attribution 4.0 International License, which permits use, sharing, adaptation, distribution and reproduction in any medium or format, as long as you give appropriate credit to the original author(s) and the source, provide a link to the Creative Commons licence, and indicate if changes were made. The images or other third party material in this article are included in the article's Creative Commons licence, unless indicated otherwise in a credit line to the material. If material is not included in the article's Creative Commons licence and your intended use is not permitted by statutory regulation or exceeds the permitted use, you will need to obtain permission directly from the copyright holder. To view a copy of this licence, visit http://creativecommonshorg/licenses/by/4.0/.

\section{References}

1. Andersen, T., Haugen, H.K., Hotop, H.: Binding energies in atomic negative ions: III. J. Phys. Chem. Ref. Data 28(6), 1511-1533 (1999)

2. Middleton, R.: A versatile high intensity negative ion source. Nucl. Instr. Methods 214, 139-150 (1983)

3. Bae, Y.K., Peterson, J.R.: Observation of the metastable negative beryllium ion, Be ${ }^{-}$. Phys. Rev. A 30, 2145-2147 (1984)

4. von Hahn, R. et al.: The cryogenic storage ring CSR. Rev. Sci. Instrum. 87, 063115 (2016)

5. Thomas, R.D. et al.: The double electrostatic ion ring experiment: a unique cryogenic electrostatic storage ring for merged ion-beams studies. Rev. Sci. Instrum. 82, 065112 (2011)

6. Forstner, O., Andersson, P., Hanstorp, D., et al.: The ILIAS project for selective isobar suppression by laser photodetachment. Nucl. Instr. Methods, B 361, 217-221 (2015)

7. Stiebing, K.E., Alexandrov, V., Dörner, R., et al.: FLSR - The Frankfurt Low energy storage ring. Nucl. Instr. Methods A 614, 10-16 (2010)

8. Pedersen, U.V., Hyde, M., MøLler, S.P., Andersen, T.: Lifetime measurement of $\mathrm{He}^{-}$utilizing an electrostatic ion storage ring. Phys. Rev. A 64, 012503 (2001)

Publisher's note Springer Nature remains neutral with regard to jurisdictional claims in published maps and institutional affiliations. 